\title{
3D Black-Blood Luminal Angiography Derived from High-Resolution MR Vessel Wall Imaging in Detecting MCA Stenosis: A Preliminary Study
}

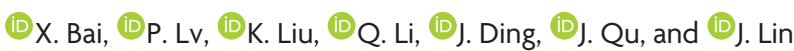

\begin{abstract}
BACKGROUND AND PURPOSE: 3D high-resolution vessel wall imaging is increasingly used for intracranial arterial diseases. This study compared the diagnostic performance of black-blood luminal angiography derived from 3D vessel wall imaging with source images of vessel wall imaging and TOF-MRA in detecting middle cerebral artery stenosis.
\end{abstract}

MATERIALS AND METHODS: Sixty-two patients with suspected MCA atherosclerosis underwent TOF-MRA, vessel wall imaging, and CTA. Intracranial black-blood luminal angiography was created from source images of vessel wall imaging using minimum intensity projection. The degree and length of MCA stenosis were measured on source images of vessel wall imaging, TOF-MRA, and black-blood luminal angiography and compared using CTA as a reference standard.

RESULTS: The image quality of black-blood luminal angiography was diagnostic in most patients. The intra- and interobserver agreement for both stenosis degree and length measurements was excellent for black-blood luminal angiography. It was comparable with that of source images of vessel wall imaging in grading stenosis. Compared with TOF-MRA, black-blood luminal angiography showed significantly higher sensitivity for the detection of severe stenosis ( $89.3 \%$ versus $64.3 \%, P=.039$ ) and higher specificity for the detection of occlusion $(95.4 \%$ versus $84.6 \%, P=.039)$. Lesion length estimated on source images of vessel wall imaging was significantly greater than that measured by CTA and black-blood luminal angiography $(P<.001$ and $P=.010)$.

CONCLUSIONS: Black-blood luminal angiography is better than TOF-MRA in detecting severe stenosis and occlusion of the MCA. Compared with source images of vessel wall imaging, it is more accurate in evaluating stenosis length. Black-blood luminal angiography can be produced as a derivative from vessel wall imaging and implemented as an adjunct to vessel wall imaging and TOF-MRA without extra acquisition time.

ABBREVIATIONS: $B B L A=$ black-blood luminal angiography; $\mathrm{VWI}=$ vessel wall imaging

ntracranial atherosclerotic stenosis is an important cause of ischemic stroke and most often involves the proximal segment of the middle cerebral artery (MCA). ${ }^{1}$ Imaging evaluation of the stenosis is critical to treatment planning. Traditionally, the degree of stenosis is evaluated by luminal angiography techniques, including DSA, CTA, and MRA. DSA, although long considered the criterion standard in assessing intracranial atherosclerotic stenosis, is limited by invasiveness, ionizing radiation, and high cost. CTA has been widely used for evaluating intracranial artery ste-

\footnotetext{
Received April 1, 2018; accepted after revision July 8.

From the Department of Radiology (X.B., P.L., K.L., J.L.), Zhongshan Hospital, Fudan University and Shanghai Institute of Medical Imaging, Shanghai, China; Departments of Neurosurgery (Q.L.) and Neurology (J.D.), Zhongshan Hospital, Fudan University, Shanghai, China; and GE Healthcare (J.Q.),Shanghai, China.

Please address correspondence to Jiang Lin, MD, Department of Radiology, Zhong shan Hospital, Fudan University and Shanghai Institute of Medical Imaging, Fenglin Rd 180, Shanghai 200032, China; e-mail: lin.jiang@zs-hospital.sh.cn

http://dx.doi.org/10.3174/ajnr.A5770
}

nosis with high accuracy, ${ }^{2}$ but it also exposes patients to ionizing radiation. Both DSA and CTA use iodinated contrast material and are restricted in patients who have previous adverse reactions to iodine and in patients with impaired renal function. Time-offlight imaging is the most commonly used unenhanced MRA technique for intracranial arterial imaging; however, its major weakness is local signal loss resulting from slow and turbulent flow. ${ }^{3}$

Recently, intracranial high-resolution vessel wall imaging (VWI) has been implemented for direct depiction of the intracranial arterial wall. Beyond the features of the arterial wall, dimensions of the vessel lumen could be reliably measured on the crosssectional images of VWI. ${ }^{4-6}$ With large spatial coverage, high signal-to-noise ratio, and flexible orientation of the reformatted images, 3D VWI is often chosen to demonstrate tortuous cerebral arteries. Clear demonstration of the vessel wall requires suppression of the MR signal of the luminal spins to yield minimum or no 
signal from the flowing blood. Based on these characteristics of 3D VWI, we attempted to convert the already acquired vessel wall images, referred to as source images of VWI, into 3D black-blood luminal angiography (BBLA) using minimum intensity projection. In this study, we used CTA as the reference standard and prospectively compared BBLA with source images of VWI and with TOF-MRA for measuring both the stenosis degree and stenosis length of the MCA. Our hypothesis was that this product of 3D VWI would be superior to source images of VWI and TOFMRA in the evaluation of MCA stenosis.

\section{MATERIALS AND METHODS \\ Patients}

From February 2017 to January 2018, sixty-two consecutive patients suspected of having intracranial atherosclerotic stenosis involving the MCA were prospectively included in the study. Patients were excluded if they had contraindications to MR imaging or nonatherosclerotic intracranial diseases, such as vasculitis, Moyamoya disease, and arterial dissection, which were clinically diagnosed according to their diagnostic criteria. ${ }^{7-9}$ Patients with extracranial carotid artery stenosis of $>50 \%$ were also excluded. All patients underwent intracranial TOF-MRA and 3D VWI, followed by CTA within 1 week. Institutional review board approval for the study design was obtained, and informed consent was obtained from all patients.

\section{MR Imaging}

All examinations were performed on a 3T MR imaging system (Discovery MR750; GE Healthcare, Milwaukee, Wisconsin) with an 8-channel head coil. The MR imaging protocol included TOFMRA and high-resolution VWI. TOF-MRA was acquired first through the circle of Willis with the following parameters: TR, 23 ms; TE, $3.4 \mathrm{~ms}$; flip angle, $20^{\circ}$; FOV, $22 \times 20.7 \mathrm{~cm}$; matrix size, $320 \times 224$; slice thickness, $1.4 \mathrm{~mm}$; mean acquisition time, $5 \mathrm{~min}$ utes 8 seconds. Using TOF-MRA as a localizer, we performed pulse sequences with different image contrast weightings, including 2D FSE T2WI, 3D-Cube T1WI (GE Healthcare), and 3DCube proton-density-weighted imaging, for assessing the intracranial vessel walls. The parameters for targeted 2D FSE T2WI were the following: TR, $2150 \mathrm{~ms}$; TE, $50 \mathrm{~ms}$; flip angle, $111^{\circ}$; FOV, $10 \times 10 \mathrm{~cm}$; matrix, $384 \times 384$; slice thickness, $2 \mathrm{~mm}$; slice gap, $0.5 \mathrm{~mm}$; NEX, 12; mean acquisition time, 4 minutes 23 seconds. The parameters for 3D-Cube T1WI were as follows: TR, $600 \mathrm{~ms}$; TE, $13 \mathrm{~ms}$; FOV , $20 \times 20 \mathrm{~cm}$; matrix size, $288 \times 288$; slice thickness, $1 \mathrm{~mm}$; NEX, 0.5; mean acquisition time, 4 minutes $16 \mathrm{sec}$ onds. 3D-Cube proton-density-weighted imaging using variable flip angle refocusing pulses was performed with the following parameters: TR, $2500 \mathrm{~ms}$; TE, $25 \mathrm{~ms}$; FOV , $20 \times 20 \mathrm{~cm}$; matrix size, $288 \times 288$, slice thickness, $1 \mathrm{~mm}$; NEX, 0.5; and mean acquisition time, 5 minutes 13 seconds. 3D-Cube proton-density-weighted imaging was performed in the sagittal plane orthogonal to the proximal MCA with intravoxel dephasing for suppression of the signal from the blood. The imaging volume was centered at the circle of Willis with voxel dimensions of $0.69 \times 0.69 \times 1 \mathrm{~mm}$ and displayed with a reconstructed resolution of $0.39 \times 0.39 \times$ $0.5 \mathrm{~mm}$.

Source images from both TOF-MRA and 3D-Cube proton- density-weighted imaging were transferred to an ADW4.6 Workstation (GE Healthcare). MIP and MPR images were created from TOF images. Minimum intensity projection was used to generate BBLA from 3D-Cube proton-density-weighted imaging.

\section{CTA}

All craniocervical CTAs, from the aortic arch to the distal intracranial arteries, were performed on a 320-detector row CT system (Aquilion ONE CT scanner; Toshiba Medical Systems, Tokyo, Japan) with the following scanning parameters: tube voltage, $120 \mathrm{kV}$ (peak); tube current, 146-210 mA; slice thickness, $0.5 \mathrm{~mm}$; FOV, $22 \times 22 \mathrm{~cm}$; and matrix size, $512 \times 512$. With a power injector, $50-70 \mathrm{~mL}$ of nonionic iodinated contrast media (Ultravist 370, iopromide; Bayer HealthCare, Berlin, Germany) was injected intravenously at a rate of $5 \mathrm{~mL} / \mathrm{s}$ followed by a $30-\mathrm{mL}$ saline bolus. CT scanning was initiated using a bolus-tracking technique at the level of the aortic arch with a trigger threshold of $150 \mathrm{HU}$.

CTA raw images were reformatted on a Vitrea $\mathrm{fX}$ workstation (Version 3.10; Toshiba Medical Systems) using MIP and MPR reconstructions for stenosis evaluation.

\section{Image Analysis}

First, an experienced neuroradiologist assessed the image quality of BBLA in demonstrating the MCA. It was graded on a 3-point scale as follows ${ }^{10}: 1$, poor visualization or nondiagnostic; 2 , moderate visualization, adequate for diagnostic purposes; and 3, good, high quality for diagnostic purposes. Patients in whom image quality was grade 1 were excluded from the comparison. Second, 2 other experienced neuroradiologists independently quantified both the degree and length of the stenosis of the MCA from 3DCube proton-density-weighted images, BBLA, TOF-MRA, and CTA. All cases were reviewed in random order. The measurements were performed using electronic calipers on the workstation. For all imaging modalities, the Warfarin Aspirin Symptomatic Intracranial Disease criteria were used to measure the MCA stenosis $^{11,12}$ : luminal stenosis $=[1-($ Dstenosis $/$ Dnormal $)] \times$ $100 \%$, where Dstenosis indicates the diameter of the residual lumen at the site of the most severe degree of stenosis, and Dnormal, the diameter of the proximal normal artery. If the proximal artery was diseased, the diameter of the distal portion of the artery at its widest point could be used instead. In case of multiple stenosis in the MCA, the most severe one was chosen for evaluation. The degree of stenosis was graded as normal $(<30 \%)$, mild $(30 \%-$ $49 \%)$, moderate $(50 \%-69 \%)$, severe stenosis $(70 \%-99 \%)$, and occlusion (100\%). ${ }^{4,13}$ Arteries with stenosis of $<30 \%$ on all these modalities were excluded from analysis. ${ }^{14}$ Stenosis length was defined as the distance from the proximal-to-distal end of the stenotic artery. ${ }^{13}$ On source images of VWI, however, the lesion length could not be measured directly but was estimated by multiplying the slice thickness and the number of the slices showing the luminal stenosis in the MCA. The lesion length was not measured in cases with total occlusion. One of the 2 neuroradiologists re-evaluated the BBLA 4 weeks later to assess intraobserver agreement. For the degree and length of stenosis, the average of the 2 radiologists' measurements was used for the final analysis. 
Comparison of the degree of stenosis with source images of VWI, BBLA, and TOF-MRA with CTA

\begin{tabular}{lcccc}
\hline \multirow{2}{*}{$\begin{array}{c}\text { Stenosis Degree on } \\
\text { VWI/BBLA/TOF-MRA }\end{array}$} & $\mathbf{3} \%$ Stenosis Degree on CTA \\
\cline { 2 - 5 } & $15 / 15 / 14$ & $\mathbf{5 0 \% - 6 9 \%}$ & $\mathbf{7 0 \% - 9 9 \%}$ & $\mathbf{1 0 0 \%}$ \\
\hline $30 \%-49 \%$ & $1 / 1 / 2$ & $0 / 0 / 1$ & $0 / 0 / 0$ & $0 / 0 / 0$ \\
$50 \%-69 \%$ & $0 / 0 / 0$ & $1 / 2 / 6$ & $0 / 0 / 0$ & $0 / 0 / 0$ \\
$70 \%-99 \%$ & $0 / 0 / 0$ & $0 / 0 / 0$ & $26 / 25 / 18$ & $0 / 0 / 0$ \\
$100 \%$ & $2 / 3 / 10$ & $15 / 15 / 15$ \\
\hline
\end{tabular}
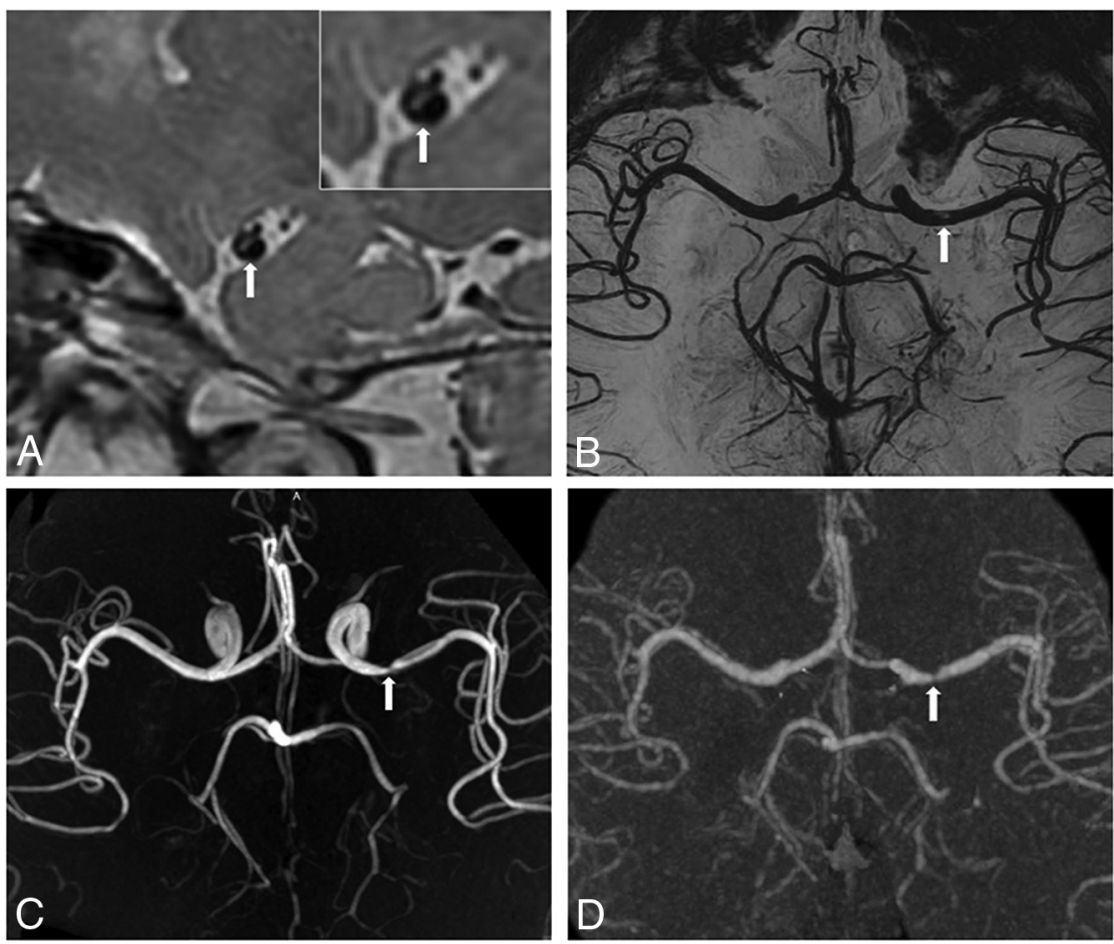

FIG 1. 3D proton-density-weighted vessel wall source image $(A)$, black-blood luminal angiography $(B)$, TOF-MRA $(C)$, and CTA $(D)$ all depict moderate stenosis of the left MI MCA (arrow). The 3D vessel wall image $(A)$ shows eccentric plaque (arrow) on the MCA wall. The inset is a magnified vessel wall image. These images are used as source images to generate luminal angiography with a minimum intensity projection.

\section{Statistical Analysis}

Commercially available software (MedCalc for Windows, Version 18; MedCalc Software, Mariakerke, Belgium) was used for the statistical analysis. Intra- and interobserver agreement in the measurement of stenosis degree and length by BBLA was assessed using the intraclass correlation coefficient. Agreement between the source images of VWI and CTA, between BBLA and CTA, as well as between TOF-MRA and CTA for the evaluation of MCA stenosis degree was calculated with the Cohen $\kappa$ test. Sensitivity and specificity in the detection of severe stenosis and occlusion of the MCA were calculated for source images of VWI, BBLA, and TOF-MRA using CTA as the reference standard. A Wilcoxon matched pairs test was performed to test whether stenosis lengths obtained from different imaging modalities were statistically different. The strength of agreement of the $\kappa$ and intraclass correlation coefficient was categorized as follows: poor, $<0.20$; fair, 0.21-0.40; moderate, 0.41-0.60; good, 0.61-0.80; and excellent, $0.81-1.00 .^{5}$ A 2-tailed $P$ value $<.05$ was considered indicative of a significant difference.

\section{RESULTS}

The image quality of BBLA in demonstrating the MCA was graded as good in 33 patients and moderate in 27 patients. Two of $62(3.2 \%)$ patients were excluded because of poor image quality from motion artifacts. A total of 80 diseased middle cerebral arteries from 60 patients (20 patients had bilateral MCA lesions) were further examined in this study. Among these patients, there were 33 men (55\%) and 27 women (45\%) with a mean age of 61.3 years (range, $45-80$ years). Thirty-nine patients $(65 \%)$ had ischemic stroke or transient ischemic attack with hemiparesis, language disorder, or perceptual deficits. The remaining 21 patients (35\%) presented with dizziness or headache.

The intra- and interobserver agreement for both stenosis degree (intraclass correlation coefficient $=0.982-0.929$, respectively) and length measurements (intraclass correlation coefficient = 0.953-0.939, respectively) were excellent for BBLA.

The Table summarizes the results of MCA stenosis degree measured with source images of VWI, BBLA, and TOFMRA in comparison with CTA. For stenosis degree, there was excellent agreement between source images of VWI and CTA $(\kappa=0.956$; 95\% CI, 0.9130.998), excellent agreement between BBLA and CTA $(\kappa=0.934 ; 95 \%$ CI, $0.882-0.986)$, and good agreement between TOF-MRA and CTA $(\kappa=0.800$; 95\% CI, 0.717-0.883) (Fig 1). Sensitivity and specificity in detecting severe stenosis were $92.9 \%$ and $98.1 \%$ with source images of VWI, $89.3 \%$ and $96.2 \%$ with BBLA, and $64.3 \%$ and $88.5 \%$ with TOF-MRA. Sensitivity and specificity in detecting occlusion were $100 \%$ and $96.9 \%$ with source images of VWI, $100 \%$ and $95.4 \%$ with BBLA, and $100 \%$ and $84.6 \%$ with TOF-MRA. The sensitivity and specificity of source images of VWI and BBLA for the detection of severe stenosis or occlusion are comparable (sensitivity, $P=.500$ and $P=1.000$; specificity, $P=1.000$ and $P=.500$ ). Compared with TOF-MRA, BBLA showed significantly higher sensitivity for the detection of severe stenosis ( $89.3 \%$ versus $64.3 \%, P=.039)$ and higher specificity for the detection of occlusion ( $95.4 \%$ versus $84.6 \%, P=.039$ ). BBLA demonstrated arteries distal to severe stenosis and occlusion in 11 cases in which TOF-MRA did not (Fig 2). The average length of the stenosis was $4.25 \pm 1.58 \mathrm{~mm}$ estimated with source images of VWI, $4.10 \pm 1.48 \mathrm{~mm}$ measured on BBLA, $4.11 \pm 1.65 \mathrm{~mm}$ on TOF-MRA, and $3.97 \pm 1.63 \mathrm{~mm}$ on CTA. The measurement of stenosis length between BBLA and CTA $(P=.060)$ and between TOF-MRA and CTA $(P=.054)$ was not significantly different. 

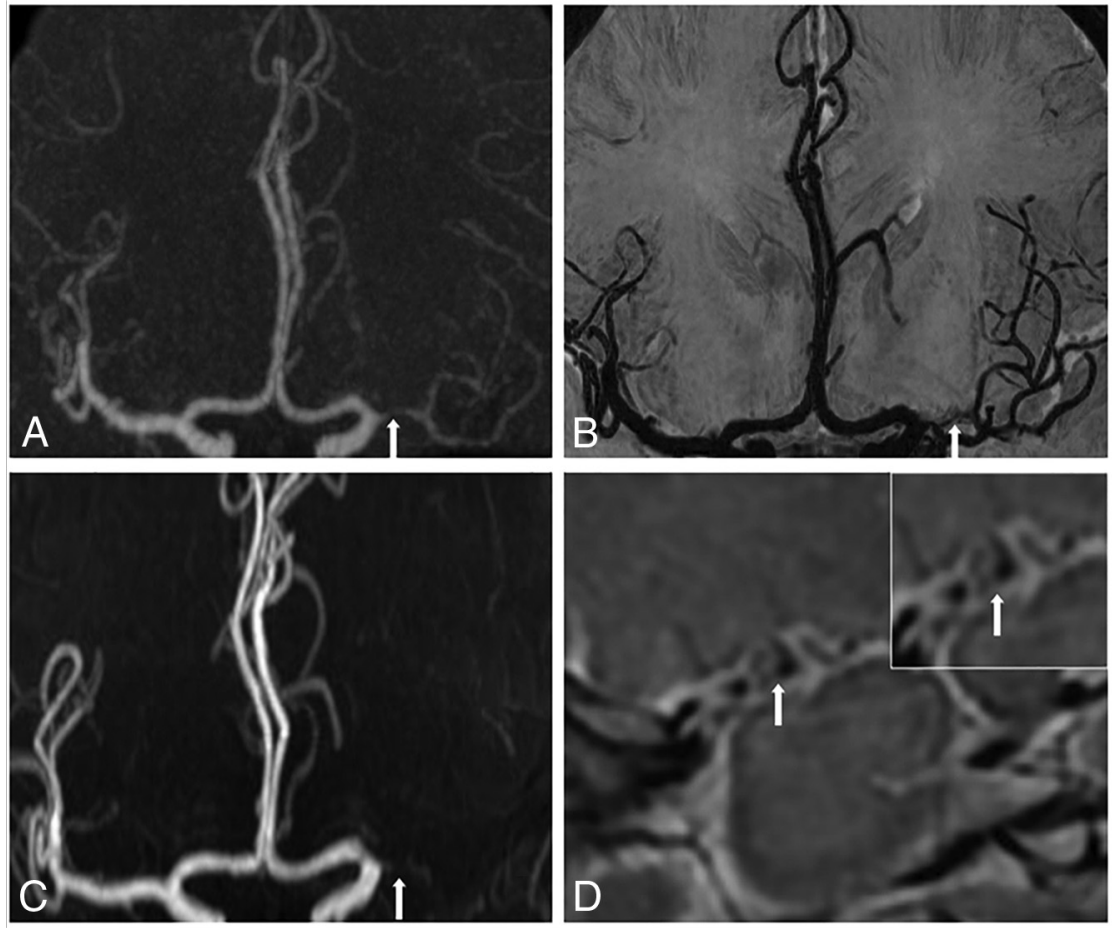

FIG 2. CTA $(A)$ and black-blood luminal angiography $(B)$ depict severe stenosis of the left MI MCA (arrow) and arteries distal to the stenosis. C, TOF-MRA overestimates this severe stenosis as an occlusion (arrow) and fails to show the distal arteries. $D$, The 3D vessel wall image shows severe stenosis of the MCA and eccentric plaque (arrow) on the vessel wall.
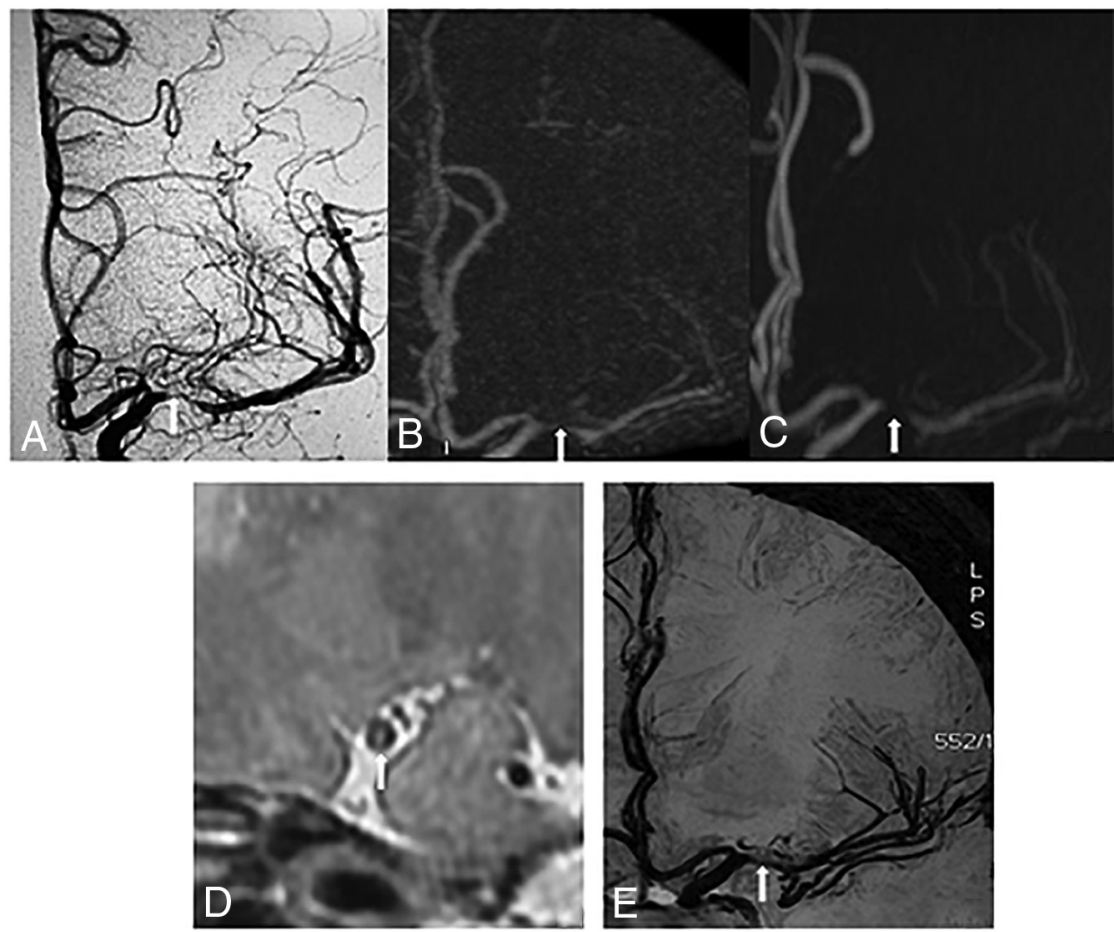

FIG 3. DSA $(A)$ and CTA (B) depict severe stenosis of the left M1 MCA (arrow). C, TOF-MRA overestimates this severe stenosis as an occlusion (arrow). Both the 3D vessel wall image $(D)$ and black-blood luminal angiography $(E)$ depict this severe stenosis correctly.

However, compared with CTA and BBLA, the estimated stenosis length was significantly larger with source images of VWI $(P<$ .001 and $P=.010$, respectively).
Twelve of 60 patients underwent DSA before possible interventional angioplasty. Fourteen diseased MCAs were identified on DSA. They were 9 occlusions, 3 severe stenoses, 1 moderate stenosis, and 1 mild stenosis. CTA, BBLA, and source images of VWI correctly diagnosed all of the 14 diseased MCAs (Fig 3) compared with DSA.

\section{DISCUSSION}

The current common practice for delineating the intracranial vessel wall is VWI; the volumetric proton intensityweighted images obtained from VWI were used as source images in this study. With the application of minimum intensity projection to these source images, we further obtained BBLA, which provided additional advantages compared with VWI alone. Our study demonstrates that the presentation of stenosis degree and length of the MCA on BBLA was in excellent agreement with the observations on CTA, which supported the high diagnostic quality of BBLA. Compared with source images of VWI, with comparable performance in the grading of stenosis, BBLA was noticeably more accurate in the measurement of stenosis length. We also found that BBLA was superior to TOF-MRA in terms of its higher sensitivity for the detection of severe stenosis and higher specificity for occlusion of the MCA. Furthermore, since BBLA did not take any additional acquisition time, we recommend its use as an adjunct to VWI and TOF-MRA in the evaluation of MCA stenosis during a single session of intracranial VWI.

In addition to its accuracy as source images of VWI in the grading of stenosis, BBLA also showed the capacity to present combined information from both VWI and MRA. By selectively suppressing the signal from blood flow, 3D black-blood MRA has been used for the assessment of vessel lumens with medium-to-large diameters, such as the carotid artery. ${ }^{15,16}$ With technical development, the application of 3D blackblood MRA has been extended to arteries of smaller diameters such as small intracranial arteries. ${ }^{17,18}$ Despite the improved resolution in luminal imaging, 3D black-blood MRA cannot provide sufficient information on the vessel wall. However, 2D/3D high-resolution 
VWI is a sequence specifically tailored to the intracranial vessel walls by sampling the cross-sections of the target vessels. Another application of VWI is that it can evaluate the luminal stenosis of the MCA with high accuracy, as already reported by some earlier studies. ${ }^{4,5,19}$ Liu et $\mathrm{al}^{4}$ reported a significant correlation between 2D VWI and DSA for the detection of MCA stenosis (>50\%) and occlusion, but stenosis length was not evaluated in that study.

Until now, no studies have yet reported the use of VWI for evaluating MCA stenosis length, which is also a critical parameter for potential interventional treatment. It is well-known from previous studies of CTA and MRA that multiple postprocessing techniques can provide an overview of the entire target vessel in a much more straightforward manner than the cross-sectional source images. ${ }^{20,21}$ Similarly, source VWI provides only the 2D cross-sectional profile of a vessel, making it challenging to form a full picture of the diseased vessel based on individual source images. Without a direct measurement of the stenosis length, however, an intuitive estimation from VWI source images is inaccurate. As shown in this study, this estimation was significantly greater than the results obtained from CTA or BBLA. In contrast, BBLA shows the entire MCA lumen from different perspectives, whereby both the degree and the length of the stenosis can be evaluated easily and accurately. More important, BBLA can be presented like conventional TOF-MRA, CTA, and DSA, with which our clinical colleagues are already familiar.

3D TOF-MRA is conventionally used for detecting luminal narrowing and serves as a localizer for subsequent VWI. However, its imaging quality depends on the prominent inflow effect of blood spins. It has intravascular signal loss at the stenosis, where blood flow is slow and turbulent, especially in small intracranial arteries. Therefore, to accurately define the stenosis, especially more severe ones, additional gadolinium-enhanced MRA or CTA is sometimes required. ${ }^{14}$ In this study, TOF-MRA was found to be less accurate than BBLA in detecting and differentiating severe stenosis and occlusion. We recorded 18 cases of overestimation of the stenosis when TOF-MRA was used, compared with 6 when BBLA was used. Furthermore, BBLA was superior to TOF-MRA in the demonstration of arteries or collaterals distal to the severe stenosis or occlusion, which is crucial for clinical intervention. Nevertheless, BBLA still overestimated stenoses in 6 cases, while source images of VWI overestimated stenoses in 4 cases in this study. The possible contributing factors were the suboptimal suppression of the blood signal and the anisotropic resolution in our reconstructed luminal images.

This study has several limitations. First, a small number of patients were enrolled, which may limit the statistical significance. Second, CTA rather than DSA was performed for comparison. However, CTA has been well-established as a highly accurate approach in identifying intracranial atherosclerotic stenosis. Using 16-detector row CT, a prior study reported that CTA performed very well compared with DSA for the detection of $\geq 50 \%$ intracranial stenosis with $97.1 \%$ sensitivity and $99.5 \%$ specificity. ${ }^{2}$ In another earlier study with a 4 -detector row technique, Bash et $\mathrm{al}^{14}$ found that CTA had a higher sensitivity and positive predictive value than TOF-MRA for the detection of intracranial stenosis and occlusion. Compared with DSA, which was available in 12 patients in our study, CTA provided identical information on
MCA stenosis. Accordingly, invasive DSA is now largely reserved for use before interventional treatment rather than for diagnosis alone. Third, our study was based on a single vascular pathology (ie, MCA stenosis). Although the MCA is the most frequently involved intracranial artery in stroke, the value of BBLA in other diseased vessel territories warrants further investigation. Fourth, owing to technical restrictions, 3D-Cube proton-density VWI was acquired with an anisotropic resolution, which may impair the demonstration of stenosis on reformatted BBLA.

\section{CONCLUSIONS}

BBLA is comparable with CTA in the evaluation of stenosis degree and the length of the MCA. It is better than TOF-MRA in detecting severe stenosis and occlusion and more accurate than VWI in measuring the stenosis length. BBLA can be produced as a derivative from VWI without extra acquisition time; therefore, it could be implemented as an adjunct to VWI and TOF-MRA.

\section{REFERENCES}

1. Weimar C, Goertler M, Harms L, et al. Distribution and outcome of symptomatic stenoses and occlusions in patients with acute cerebral ischemia. Arch Neurol 2006;63:1287-91 CrossRef Medline

2. Nguyen-Huynh MN, Wintermark M, English J, et al. How accurate is $\mathrm{CT}$ angiography in evaluating intracranial atherosclerotic disease? Stroke 2008;39:1184-88 CrossRef Medline

3. Willinek WA, Born M, Simon B, et al. Time-of-flight MR angiography: comparison of 3.0-T imaging and 1.5- $\mathrm{T}$ imaging - initial experience. Radiology 2003;229:913-20 CrossRef Medline

4. Liu Q, Huang J, Degnan AJ, et al. Comparison of high-resolution MRI with CT angiography and digital subtraction angiography for the evaluation of middle cerebral artery atherosclerotic steno-occlusive disease. Int J Cardiovasc Imaging 2013;29:1491-98 CrossRef Medline

5. Lee NJ, Chung MS, Jung SC, et al. Comparison of high-resolution MR imaging and digital subtraction angiography for the characterization and diagnosis of intracranial artery disease. AJNR Am JNeuroradiol 2016;37:2245-50 CrossRef Medline

6. Park JE, Jung SC, Lee SH, et al. Comparison of 3D magnetic resonance imaging and digital subtraction angiography for intracranial artery stenosis. Eur Radiol 2017;27:4737-46 CrossRef Medline

7. Mandell DM, Mossa-Basha M, Qiao Y, et al; Vessel Wall Imaging Study Group of the American Society of Neuroradiology. Intracranial vessel wall MRI: principles and expert consensus recommendations of the American Society of Neuroradiology. AJNR Am J Neuroradiol 2017;38:218-29 CrossRef Medline

8. Kim YJ, Lee DH, Kwon JY, et al. High resolution MRI difference between moyamoya disease and intracranial atherosclerosis. Eur J Neurol 2013;20:1311-18 CrossRef Medline

9. Debette S, Compter A, Labeyrie MA, et al. Epidemiology, pathophysiology, diagnosis, and management of intracranial artery dissection. Lancet Neurol 2015;14:640-54 CrossRef Medline

10. van der Kolk AG, Hendrikse J, Brundel M, et al. Multi-sequence whole-brain intracranial vessel wall imaging at 7.0 Tesla. Eur Radiol 2013;23:2996-3004 CrossRef Medline

11. Samuels OB, Joseph GJ, Lynn MJ, et al. A standardized method for measuring intracranial arterial stenosis. AJNR Am J Neuroradiol 2000;21:643-46 Medline

12. Huang J, Degnan AJ, Liu Q, et al. Comparison of NASCET and WASID criteria for the measurement of intracranial stenosis using digital subtraction and computed tomography angiography of the middle cerebral artery. J Neuroradiol 2012;39:342-45 CrossRef Medline

13. Jeon JS, Sheen SH, Hwang GJ, et al. Feasibility of intravenous flat 
panel detector CT angiography for intracranial arterial stenosis. AJNR Am J Neuroradiol 2013;34:129-34 CrossRef Medline

14. Bash S, Villablanca J, Jahan R, et al. Intracranial vascular stenosis and occlusive disease: evaluation with CT angiography, MR angiography, and digital subtraction angiography. AJNR Am J Neuroradiol 2005;26:1012-21 Medline

15. Lv P, Lin J, Guo D, et al. Detection of carotid artery stenosis: a comparison between 2 unenhanced MRAs and dual-source CTA. AJNR Am J Neuroradiol 2014;35:2360-65 CrossRef Medline

16. Zhao H, Wang J, Liu X, et al. Assessment of carotid artery atherosclerotic disease by using three-dimensional fast black-blood MR imaging: comparison with DSA. Radiology 2015;274:508-16 CrossRef Medline

17. Stivaros SM, Harris JN, Adams W, et al. Does black blood MRA have a role in the assessment of intracerebral aneurysms? Eur Radiol 2009;19:184-92 CrossRef Medline

18. Alexander AL, Buswell HR, Sun Y, et al. Intracranial black-blood MR angiography with high-resolution 3D fast spin echo. Magn Reson Med 1998;40:298-310 CrossRef Medline

19. Ryu CW, Jahng GH, Kim EJ, et al. High resolution wall and lumen MRI of the middle cerebral arteries at 3 Tesla. Cerebrovasc Dis 2009; 27:433-42 CrossRef Medline

20. Lell MM, Anders $\mathrm{K}$, Uder M, et al. New techniques in CT angiography. Radiographics 2006;26(Suppl 1):S45-62 CrossRef Medline

21. Ota H, Takase K, Rikimaru H, et al. Quantitative vascular measurements in arterial occlusive disease. Radiographics 2005;25:1141-58 CrossRef Medline 